\title{
Correlation of skin and nerve histopathology in leprosy
}

\author{
S. K. ASHOK KUMAR, B. S. N. REDDY* \\ \& C. RATNAKAR \\ Department of Dermatology \& STD and Pathology, JIPMER, \\ Pondicherry-605006, India
}

Accepted for publication 28 November 1995

\begin{abstract}
Summary Discrepancies have been noted in the histopathological findings between skin and nerve lesions of leprosy patients in some recent works. We studied concurrent skin and nerve biopsies in 27 randomly selected leprosy patients to correlate the histopathological features of skin and nerve lesions, and to assess the importance of neural histology in the classification of leprosy.

Skin and nerve biopsies were diagnostic of leprosy in 23 and 26 patients, respectively. A discrepancy was found between the two in 15 cases. Neural histology was helpf ul in the classification of determinate forms in 24 cases while dermal histology was significant only in 16 patients. A multibacillary nerve and paucibacillary skin picture was observed in 3 patients.

It was concluded that nerve biopsy is more informative and specific than skin biopsy in the diagnosis of leprosy and further helps to classify the patients when the skin histology is inderminate or nonspecific.
\end{abstract}

\section{Introduction}

At present the diagnosis and classification of leprosy is largely based on the characteristic skin lesions, thickened peripheral nerves and the presence of anaesthesia. Demonstration of AFB in slit-skin smears and histopathology of the skin are the commonly-used methods to confirm its diagnosis and classification. Although these criteria are related to the skin parameters, leprosy is primarily a disease of peripheral nerves. ${ }^{1}$

Several discrepancies have been noted in the histopathological features of skin and nerve lesions in leprosy. Some authors observed a higher bacterial load and lower histological grading of the disease in biopsies obtained from the peripheral nerves than from skin. ${ }^{2-8}$ These cases, if diagnosed and treated on the basis of skin findings alone, may result in inadequate treatment, thereby contributing towards increased drug

* Correspondence: Professor \& Head of Department of Dermatology \& STD, Maulana Azad Medical College, New Delhi-2 India. 
resistance and relapse of the disease. In contrast, no such discordance was noted in the histopathology of skin and nerve lesions by some other workers. ${ }^{9,10}$ The reasons for this anomaly are far from clear and no explanation was accounted. Hence, the present study was carried out to correlate the histopathology of the skin and nerve lesions in concurrent biopsies obtained from different types of leprosy patients and to assess the role of neural histology in the classification of disease.

\section{Material and methods}

The material for this study consisted of 27 randomly selected different types of leprosy patients reported to our urban leprosy clinic. They were in the age group of 7-60 years, comprising 21 males and 6 females. The duration of the disease varied from 2 weeks to 10 years. Among these, 20 were new cases without any treatment, while 7 had completed the course of paucibacillary treatment but the disease was still active.

A provisional diagnosis of leprosy was made on clinical criteria according to RidleyJopling classification ${ }^{1}$ in all cases except the pure neuritic, which were diagnosed as per Indian Classification. ${ }^{11}$ These included TT (1), BT (20), BB (1), BL (2), LL (1) and pure neuritic (2). Slit-skin smears for AFB and lepromin test (Lepromin-A) were done and all patients underwent parallel skin and nerve biopsies. Skin biopsies were obtained from the suggestive lesion and from the most hypoesthetic part in pure neuritic cases. Nerve biopsies were taken from pure sensory cutaneous nerves such as radial cutaneous, sural, superficial peroneal or cutaneous nerve of forearm.

The histopathological features were studied in H. \& E. stained sections and a bacillary count for AFB was carried out on a logarithmic scale in sections stained with modified Fite-Faraco method. The skin biopsies were classified according to Ridley-Jopling, as described by Ridley. ${ }^{12}$

Indeterminate leprosy: there is no granuloma present but one or more of the following features are seen: a, Infiltration of lymphocytes and histiocytes around skin appendages, nerves and vessels, with or without proliferation of spindle shaped cells in the superficial dermis; b, proliferation of Schwann cells; or c, AFB in nerve, arrector pili muscle or subepidermal zone.

The histopathological criteria laid down by Ridley \& Ridley ${ }^{13}$ were used for the evaluation of nerve biopsies. Further, the histological diagnosis of indeterminate leprosy and leprous neuritis in the nerve was made according to Job ${ }^{14}$ based on either (a) presence of bacill in Schwann cells with the nerve almost looking normal without any damage to the structure, or (b) the perineurium showing some reactive proliferation with infiltration and collection of monocular cell around neurovascular bundles. The nerve parenchyma may be completely destroyed and replaced by hyalinized fibrous tissue with hardly any inflammatory cells in cases of long-standing leprous neuritis (chronic leprosy neuritis).

\section{Results}

CLINICOPATHOLOGIC CORRELATION

Skin histology was diagnostic of leprosy in 23 out of 27 patients studied comprising 
Table 1. Clinicopathological correlation (skin)

\begin{tabular}{lccccccr}
\hline $\begin{array}{l}\text { Histological } \\
\begin{array}{l}\text { diagnosis } \\
\text { (skin) }\end{array}\end{array}$ & TT & BT & BB & BL & LL & PN* & Total \\
\cline { 2 - 6 } & 1 & - & - & - & - & - & 1 \\
TT(s) & - & 11 & - & - & - & - & 11 \\
BT & - & - & 1 & 1 & - & - & 2 \\
BB & - & - & - & 1 & - & - & 1 \\
BL & - & - & - & - & 1 & - & 1 \\
LL & - & 7 & - & - & - & - & 7 \\
I Non-Specific & - & 2 & - & - & - & 2 & 4 \\
Total & 1 & 20 & 1 & 2 & 1 & 2 & 27 \\
\hline
\end{tabular}

* Pure neuritic leprosy (skin biopsy done from hypoesthetic part).

indeterminate (7), $\mathrm{TT}_{\mathrm{S}}$ (1), BT (11), BB (2), BL (1) and LL (1), and the picture was non-specific in 4 cases. Clinicopathological correlation with reference to dermal histology and clinical diagnosis was seen in 15 out of 27 cases studied $(56 \%)$ as shown in Table 1.

Neural histology was diagnostic of Hansen's disease in 26 out of 27 cases studied constituting indeterminate (1), TT (6), BT (11), B (2), BL (3), LL (2), leprous neuritis (1) and normal picture in one patient. Clinicopathological correlation with reference to the clinical diagnosis and neural histology was noted in 14 out of 27 cases $(52 \%)$ as shown in Table 2. In BT group concordance was seen in 10 out of 20 patients, followed by one each in TT, BB, BL and LL.

\section{CORRELATION OF SKIN AND NEURAL HISTOLOGY}

Histopathology of skin and nerve correlated in 7 BT cases followed by one case each in

Table 2. Clinicopathological correlation (nerve)

\begin{tabular}{|c|c|c|c|c|c|c|c|}
\hline \multirow{2}{*}{$\begin{array}{l}\text { Histological } \\
\text { diagnosis } \\
\text { (nerve) }\end{array}$} & \multicolumn{6}{|c|}{ Clinical diagnosis } & \multirow[b]{2}{*}{ Total } \\
\hline & TT & BT & $\mathrm{BB}$ & $\mathrm{BL}$ & LL & PN* & \\
\hline TTs & 1 & 4 & - & - & - & 1 & 6 \\
\hline BT & - & 10 & - & - & - & 1 & 11 \\
\hline $\mathrm{BB}$ & - & 1 & 1 & - & - & - & 2 \\
\hline $\mathrm{BL}$ & - & 2 & - & 1 & - & - & 3 \\
\hline $\mathrm{LL}$ & - & - & - & 1 & 1 & - & 2 \\
\hline I & - & 1 & - & - & - & - & 1 \\
\hline Chronic leprous & & & & & & & \\
\hline neuritis & - & 1 & - & - & - & - & 1 \\
\hline Normal & - & 1 & - & - & - & - & 1 \\
\hline Total & 1 & 20 & 1 & 2 & 1 & 2 & 27 \\
\hline
\end{tabular}

* Pure neuritic. 
Table 3. Correlation of skin and nerve histopathology

\begin{tabular}{lcccccccr}
\hline & \multicolumn{7}{c}{ Skin pathology } \\
\cline { 2 - 7 } $\begin{array}{l}\text { Nerve } \\
\text { pathology }\end{array}$ & TT & BT & BB & BL & LL & I & Others & Total \\
\hline TT & 1 & 3 & - & - & - & 1 & 1 & 6 \\
BT & - & 7 & - & - & - & 2 & 2 & 11 \\
BB & - & - & 1 & - & - & 1 & - & 2 \\
BL & - & 1 & 1 & - & - & 1 & - & 3 \\
LL & - & - & - & 1 & 1 & - & - & 1 \\
Indeterminate & - & - & - & - & - & 1 & - & 2 \\
Others & - & - & - & - & - & 1 & 1 & 27 \\
Total & 1 & 11 & 2 & 1 & 1 & 7 & 4 & 27 \\
\hline
\end{tabular}

* Others include nonspecific histology and leprous neuritis (leprous neuritis diagnostic but not classifiable, so included in others).

TT, BB, LL, indeterminate and others (Table 3). Overall concordance was noted in 12 out of 27 cases $(44 \%)$.

The neural histology was helpful in the classification of determinate forms (TT, BT, $\mathrm{BB}, \mathrm{BL} \& \mathrm{LL})$ in 24 patients while dermal histology was only useful in 16 patients. This difference was statistically significant $(P<0 \cdot 02)$. AFB were seen in 13 patients altogether, nerve alone (6), skin alone (2) and both nerve and skin (5) patients. Regarding the bacteriologic index (BI) in the biopsies, a multibacillary (MB) picture was seen in 11 nerve and 7 skin biopsies, thereby revealing that nerve lesions had higher bacterial index than concurrent skin lesions (Table 4).

Caseation necrosis was considered to be more indicative of subpolar tuberculoid (TTs) by Ridley. ${ }^{15}$ This feature was observed in 6 nerve biopsies and one skin biopsy in our series. Since, we followed the criteria laid out in the nerve biopsy classification of Ridley \& Ridley, ${ }^{13}$ these cases were included under TTs. The data pertaining to the

Table 4. Bacteriologic index in skin and nerve biopsies

\begin{tabular}{|c|c|c|c|c|c|c|c|}
\hline \multirow[b]{2}{*}{ Skin biopsy } & \multirow{2}{*}{$\begin{array}{l}\text { No. of } \\
\text { cases }\end{array}$} & \multicolumn{2}{|c|}{$\begin{array}{l}\text { Bacteriologic index } \\
\text { (BI) }\end{array}$} & \multirow[b]{2}{*}{ Nerve biopsy } & \multirow{2}{*}{$\begin{array}{l}\text { No. of } \\
\text { cases }\end{array}$} & \multicolumn{2}{|c|}{$\begin{array}{l}\text { Bacteriologic index } \\
\text { (BI) }\end{array}$} \\
\hline & & PB & MB & & & PB & $\mathrm{MB}$ \\
\hline TTs & 1 & - & 1 & TTs & 6 & 4 & 2 \\
\hline BT & 11 & 9 & 2 & BT & 11 & 9 & 2 \\
\hline $\mathrm{BB}$ & 2 & - & 2 & $\mathrm{BB}$ & 2 & - & 2 \\
\hline $\mathrm{BL}$ & 1 & - & 1 & $\mathrm{BL}$ & 3 & - & 3 \\
\hline $\mathrm{LL}$ & 1 & - & 1 & $\mathrm{LL}$ & 2 & - & 2 \\
\hline Indeterminate & 7 & 7 & - & Indeterminate & 1 & 1 & - \\
\hline \multirow[t]{2}{*}{ Nonspecific } & 4 & 4 & - & Leprous neuritis & 1 & 1 & - \\
\hline & & & & Normal picture & 1 & 1 & - \\
\hline Total & 27 & 20 & 7 & & 27 & 16 & 11 \\
\hline
\end{tabular}

$\mathrm{PB}$ means $\mathrm{BI}=0 . \mathrm{MB}$ means $\mathrm{BI}=\geqslant 1+$. 
Table 5. Immunological (Mitsuda response) and histological correlation of skin and nerve

\begin{tabular}{lcccc}
\hline & \multicolumn{4}{c}{ Mitsuda reaction } \\
\cline { 2 - 5 } Histological diagnosis & - ve & $1+$ & $2+$ & $3+$ \\
\hline Skin & - & - & - & 1 \\
TT & 1 & 2 & 3 & 5 \\
BT & 2 & - & - & - \\
BB & 1 & - & - & - \\
BL & 1 & - & - & - \\
LL & 2 & 2 & 2 & 1 \\
Indeterminate & - & 2 & - & 2 \\
Nonspecific & & & & \\
Nerve & - & - & 1 & 5 \\
TT & - & 4 & 4 & 3 \\
BT & 2 & - & - & - \\
BB & 3 & - & - & - \\
BL & 2 & - & - & - \\
LL & - & 1 & - & - \\
Indeterminate & - & - & - & - \\
Chronic leprous neuritis & - & 1 & - & - \\
Normal & & & &
\end{tabular}

immunohistopathological correlation (Lepromin response) with reference to the nerve and skin were summarized in Table 5.

\section{Discussion}

No doubt, the Ridley-Jopling classification ${ }^{1}$ is widely accepted, but its histological component refers only to skin with the presumption that there may be no significant difference in the classification of skin and neural histology. However, discrepancies between skin and nerve lesions were noted in the form of increased number of bacilli and a lower, i.e. towards LL, histological grading in nerves than in skin. Srinivasan et al. ${ }^{3}$ found discrepancies in 21 out of 36 cases which included 6 cases of pure neuritic leprosy. The nerves showed a lower histological grading in most of the cases. ${ }^{8,13,16,17}$ We observed discordance between skin and nerve histology in 15 out of 27 patients. The discrepancy in the form of lower histological grading occurring in nerves was observed in 3 cases, i.e. paucibacillary skin and multibacillary nerve.

Ridley et al. ${ }^{18}$ attributed the discrepancy between skin and nerve in the histological grading to the microreactions occurring in the peripheral nerves. They described these reactions as clinically silent and regular features of peripheral nerve involvement. These hypersensitivity reactions, occurring in nerves alone can be either upgrading or downgrading and can lead to alterations in the classification of nerve lesions locally, without causing much difference in the skin.

The importance of neural histology in the classification of leprosy is less well documented. Mukherjee \& Mishra ${ }^{16}$ found neural histology to be more useful in the classification of the disease in one third of their patients in whom skin showed 
indeterminate or nonspecific features. Srinivasan et al. ${ }^{3}$ noted that the lesion in the nerve was classifiable in 9 patients while the skin lesion in the same patients could be identified as leprosy but not, classifiable as any particular type. Kaur et al. ${ }^{8}$ also found nerve histology is more significant in the classification of leprosy. Our findings further confirm this point.

The neural histology was helpful in the classification of determinate forms (TT, BT, $\mathrm{BB}, \mathrm{BL}$ or LL) in 24 out of 27 patients studied in our series while the corresponding dermal histology was significant only in 16 patients. This difference was statistically significant $(p<0 \cdot 02)$. Skin showed features of indeterminate leprosy or nonspecific changes in 11 cases while the corresponding lesion in the nerve showed granuloma classifiable as TT (2), BT (4), BB (1) and BL (1) in 8 out of 11 cases.

The relevance of lower immunological grading and higher bacterial load in the nerves is debated. Nerves are protected sites for Mycobacterium leprae and allow unhindered multiplication of bacilli in the early stages of infection. Kaur et al. ${ }^{8}$ and Negesse et al. ${ }^{19}$ described that such patients should be classified as multibacillary on the basis of combined skin and neural histology and treated with MB therapy to prevent relapse and resistance to drugs. We believe that it is an important and logical consideration.

The discrepancy in the skin and nerve bacillary load was explained by Ridley \& Ridley, ${ }^{13}$ on the basis of delayed recognition of $M$. leprae antigen in the nerve, leading to increased load of bacilli. They also argued that skin harbours the main mass of $M$. leprae in the body and hence the skin classification rather than the nerve, represents the general tissue response. But Negesse et al. ${ }^{19}$ observed that the patients with MB nerve lesions have low lymphoproliferative assay response regardless of their BI in skin lesions. Hence, they suggested that bacillary load in the nerve is certainly one of the factors in determining the immunological spectrum of the disease. Srinivasan et al. ${ }^{3}$ showed that 7 out of 8 patients with clinical relapse displayed relapse histopathologically only in nerves. These results are in contrast to the view expressed by Ridley \& Ridley ${ }^{13}$ that the skin tissue response reflects the general immune status. Our data pertaining to the immunopathological (both skin and nerve) correlation based on Mitsuda reaction using lepromin-A were within established norms. Mitsuda was negative in indeterminate (2) and BT (1) cases based on skin histology. These were found to be BB or BL on neural histology. Nerve pathology correlated more exactly with Mitsuda reaction; TT (5) showed strong Mitsuda reaction with ulceration in 4 of them. BG showed varying picture $(1+$ to $3+)$ while $\mathrm{BB}, \mathrm{BL}$ and LL showed negative reaction. However, Srinivasan et al. $^{3}$ have observed positive Mitsuda in a case in whom nerve biopsy showed BL features. Kaur et al. ${ }^{21}$ have also described this in some of their cases.

The role of nerve biopsy in the diagnosis of pure neuritic leprosy is well established. Thirty-eight out of 77 patients with primary neuritic leprosy were confirmed to have leprosy on the basis of nerve biopsy in a study done by Jacob \& Mathai. ${ }^{20}$ Similar role of nerve biopsy in the diagnosis of leprosy was observed by Kaur et al. ${ }^{21}$ In our study the diagnosis of leprosy was possible in both pure neuritic cases in nerve biopsy, while normal looking hypoesthetic skin did not show evidence of leprosy. Similarly, no significant pathology was found in the analgesic skin of 39 pure neuritic cases studied by Kaur et $a l^{21}$ Nevertheless, Pannikar et $a l^{22}$ found skin biopsy showing features of leprosy even in the absence of skin lesion in 14 out of 27 pure neuritic cases.

$M$. leprae continue in a viable state in certain sites like peripheral nerves long after they are cleared from the skin. These organisms have been considered as persisters. They 
play a vital role in the relapse of the disease. In the present study clinical relapse was seen in 7 cases. Neural histology in these patients showed granuloma classifiable as BT or TT in 5 cases, while skin histology showed classifiable leprosy in only 3 cases. Srinivasan et $\mathrm{al}^{3}$ found the evidence of relapse occurring only in nerve lesions in 7 out of 8 cases without corresponding evidence in the skin biopsy. Most of these cases showed lepromatous picture. Hence, it is important to keep the persisters in view before considering the patients for release from treatment.

Discrepancy between skin and nerve histology is significant especially in the classification of the disease, with nerve histology adds detail to the evaluation of patient and in turn, we might understand how it reflects the immune response of the patient.

\section{References}

${ }^{1}$ Ridley DS, Jopling WH. Classification of leprosy according to immunity. A five group system. Int J Lepr, 1966; 34: 255-273.

2 Antia NH, Pandya NJ. Qualitative histology and quantitative bacteriology in various tissues of 50 leprosy patients. Lepr Rev, 1976; 47: 175-183.

3 Srinivasan H, Rao KS, Iyer CAS. Discrepancy in the histological features of leprosy lesions in the skin and peripheral nerve. Le pr India, 1982; 54: 275-286.

4 Halmanot RT, Mahan RN, McDougall AC, Anderson JG. Sural nerve biopsy in leprosy patients after varying periods of treatment, histological and bacteriological findings in light microscopy. Int J Le pr, 1984, 52: $163-170$.

5 Nilsen R, Mengistu G, Reddy BB. The role of nerve biopsies in the diagnosis and management of leprosy. Lepr Rev, 1989; 60: 28-32.

6 Pereira JH, Palande DD, Meissner SE. Mycobacteria in nerve trunks of long term treated leprosy patients. Lepr Rev, 1991; 63: 131-142.

${ }^{7}$ Shetty VP, Suchitra K, Uplekar MW, Antia NH. Persistence of M. leprae in the peripheral nerve as compared to the skin of multidrug-treated leprosy and its role in the classification of leprosy. Lepr Rev, 1992; 63: 329-336.

${ }^{8}$ Kaur S, Sharma VK, Basak P, Kaur I, Radotra BD. Concurrent skin and nerve histology in leprosy and its role in classification of leprosy. Lepr Rev, 1993; 64: 110-115.

9 Pedley JC, Harman DJ, Waudby H, McDougal AC. Leprosy in peripheral nerves histological findings in the 119 untreated patients in Nepal. J Neurol Neurosurg Psychiatry, 1980; 43: 198-204.

10 Liu-Tze-Chun, Qui Ju-Shi. Pathological findings in the peripheral nerves, lymph nodes and visceral organs of leprosy. Int J Lepr, 1984; 52: 377-383.

11 Indian classification (new). Clinical, Histopathological and Immunological features of the five-types classification approved by the Indian Association of Leprologists. Lepr India, 1982; 54: 22-32.

12 Ridley DS. Skin biopsy in leprosy. Documenta Geigy. 3rd Edn. Basel: Ciba-Geigy, 1990.

13 Ridley DS, Ridley MJ. Classification of nerves is modified by the delayed recognition of M. leprae. Int J Lepr, 1986; 54: 596-605.

14 Job CK. Nerve damage in leprosy. Int J Lepr, 1989; 57: 532-539.

15 Ridley DS. Pathogenesis of leprosy and related diseases. London, Wright, 1988: 168-171.

16 Mukher jee A, Mishra RS. Comparative histology of skin and nerve granulomas in leprosy patients. Lepr $J$ Rev, 1988; 59: 177-180.

17 Chacko CJG. Evolution of pathological lesions in leprosy patients and their immunological significance. Proceedings of the Indo-UK Symposium in leprosy. Agra, India. April 1986: 82-89.

18 Ridley MJ, Waters MFR, Ridley DS. Effect of $M$. leprae in peripheral nerve on the evolution of skin lesion. Int J Lepr, 1994; 62: 99-106.

19 Negesse Y, Beimnet K, Miko T, Wondimug A, Berhan TY. In leprosy the presence of mycobacteria in the nerve is an essential factor in the cycle and spectrum of Mycobacterium Le prae Inf ection. Le pr Rev, 1993; 64: 104-109.

20 Jacob M, Mathai R. Diagnostic efficacy of Cutaneous nerve biopsy in primary neuritic leprosy. Int J Lepr, 1988; 56: 56-61.

${ }^{21}$ Kaur G, Bhawaneshwar K, Girdhar K, Girdhar J, Sengupta U, Desikan KV. Clinical, immunological and histological study of neuritic leprosy patients. Int J Le pr, 1991; 59: 385-391.

22 Pannikar CK, Arunthati S, Checko CJG, Frutschi EP. A Clinico-pathological study of primary neuritic leprosy. Lepr India, 1983; 55: 212-222. 